Cahiers
de a Recherche
Fourles Droits
Fondamentaux

Cahiers de la recherche sur les droits fondamentaux

$14 \mid 2016$

Urbanisme et droits fondamentaux

\title{
Chronique de jurisprudence du droit des étrangers 2015
}

A Chronicle of the Case Law of the Rights of Aliens 2015

Guillaume Dujardin

\section{(2) OpenEdition}

Journals

Édition électronique

URL : https://journals.openedition.org/crdf/602

DOI : $10.4000 /$ crdf.602

ISSN : 2264-1246

Éditeur

Presses universitaires de Caen

Édition imprimée

Date de publication : 1 novembre 2016

Pagination : 137-146

ISBN : 978-2-84133-838-2

ISSN : 1634-8842

Référence électronique

Guillaume Dujardin, «Chronique de jurisprudence du droit des étrangers 2015 », Cahiers de la recherche sur les droits fondamentaux [En ligne], 14 | 2016, mis en ligne le 01 octobre 2019, consulté le 15

novembre 2022. URL : http://journals.openedition.org/crdf/602 ; DOI : https://doi.org/10.4000/crdf. 602

Tous droits réservés 


\title{
Chronique de jurisprudence du droit des étrangers 2015
}

\author{
Guillaume DUJARDIN \\ Doctorant en droit public à l'université de Caen Normandie \\ Centre de recherche sur les droits fondamentaux et les évolutions du droit (CRDFED, EA 2132)
}

I. Le droit commun des étrangers

A. L'expulsion d'un "fou »: prévenir l'atteinte à l'ordre public plutôt que la punir

B. L'applicabilité du Code de l'entrée et du séjour des étrangers et du droit d'asile face à un accord bilatéral : faire enfin dire à l'accord ce qu'il dit...

II. Enfin un statut de réfugié solide pour les victimes de traite

Pour cette deuxième année, et nonobstant l'actualité, la chronique a décidé de ne pas faire peau neuve. Il ne sera donc toujours pas question de traiter de l'actualité législative dans celle-ci. Cette année, comme à peu près toutes avant elle, aurait pourtant pu s'y prêter... Avec une retentissante loi réformant l'asile, le législateur a tenté de répondre à la nécessité de réduire le délai d'examen des demandes d'asile. Projets et propositions de loi continuent également de pleuvoir. Tel un serpent de mer, la protection des victimes de traite des êtres humains intéresse la proposition de loi visant à renforcer la lutte contre le système prostitutionnel. Pour ce qui nous intéresse, elle a pour but de créer, à côté du dispositif de l'article L. 316-1 du Code de l'entrée et du séjour des étrangers et du droit d'asile (CESEDA) et de la protection offerte par la Convention de Genève sur le statut de réfugié du 28 juillet 1951, une troisième voie, certes plus précaire (en raison de la courte durée du titre de séjour octroyé), mais plus facile à mettre en œuvre (car déconnectée du dépôt de plainte) ${ }^{1}$. Plus encore, le très médiatisé projet de loi constitutionnel aurait pu innover sur la possibilité de déchoir de sa nationalité un binational, condamné à titre définitif pour acte de terrorisme...

Le choix des thèmes retenus cette année n'aura pas été évident. Tellement d'arrêts auraient mérité notre attention... Le Conseil d'État a partiellement ouvert le référé-liberté pour les mesures de placement en rétention administrative $^{2}$; le feuilleton contentieux de la «jungle de Calais» a trouvé son épilogue contentieux ${ }^{3}$... Cette liste pourrait être poursuivie, et confirme le caractère un brin discrétionnaire de nos choix. N'est-ce pas là cela dit la marque de la matière... Royaume de la «discrétionnarité », celle-ci se poursuit jusque dans les thèmes choisis cette année!

Cette année marquera en tout cas une innovation dans les thèmes choisis. Pour tenir compte de l'actualité, le droit des réfugiés - avec les victimes de proxénétisme - fera son entrée (II). Mais le droit commun des étrangers occupera cette année encore la «pole position» (I).

1. Depuis l'écriture de ces quelques lignes, ladite proposition de loi a, enfin, abouti. L'article 8 de la loi no $2016-444$ du 13 avril 2016 est venu insérer dans le CESEDA un nouvel article L. 316-1-1.

2. CE, 11 juin 2015, n 390704: L'actualité juridique. Droit administratif, 2015, p. 2042, note L. Fermaud.

3. CE, ord., 23 novembre 2015, Ministre de l'Intérieur c. commune de Calais, $\mathrm{n}^{\circ} 394540$ et 394568: Revue de droit sanitaire et social, 2016 , p. 90 , note D. Roman. 


\section{Le droit commun des étrangers}

\section{A. L'expulsion d'un "fou »: prévenir l'atteinte à l'ordre public plutôt que la punir}

La France serait-elle «crispée sur son ordre public ${ }^{4}$ ? La jurisprudence française sur l'expulsion invite le juriste à se poser la question. À l'heure où l'Europe des droits de l'homme se trouve frappée dans sa chair par de terribles attentats terroristes, il est naturel pour lui de s'interroger sur les mécanismes juridiques permettant l'éloignement d'une personne, fût-elle en situation régulière, afin de prévenir que soit gravement porté atteinte à l'ordre public. L'émotion suscitée par certaines actualités est pourtant, ici aussi, le pire ennemi du juriste. La mesure d'expulsion ne vise-t-elle qu'à éloigner un étranger ayant commis une infraction grave, et où tout porte à penser qu'il se rendra de nouveau coupable d'un tel comportement gravement nuisible à la société? Ou, à l'inverse, permet-elle, en l'absence de toute infraction préalablement commise, d'empêcher un individu de «passer à l'action»?

Un arrêt rendu par le juge des référés du Conseil d'État le 7 mai 2015 a pour mérite de trancher ce débat, au moins en partie. Les faits de l'espèce se résument aisément. De nationalité algérienne, et titulaire depuis 2014 d'un certificat de séjour de dix ans en qualité d'étranger malade, M. B. a été frappé par un arrêté d'expulsion le 10 avril 2015. L'intéressé a alors saisi le juge des référés du tribunal administratif de Paris aux fins de suspendre l'exécution dudit arrêté. Par une ordonnance du 30 avril 2015, le juge des référés parisien a fait droit à cette demande, et enjoint aux autorités consulaires de prendre toutes mesures de nature à permettre son retour en France. Le ministre de l'Intérieur interjette alors appel de cette ordonnance devant le Conseil d'État.

Par un arrêt du 7 mai $2015^{5}$, la juridiction suprême casse et annule l'ordonnance du 30 avril, en considérant que:

[...] le ministre de l'intérieur pouvait légalement prendre en compte l'état de santé mental de M. B... comme un élément de nature à caractériser l'existence d'une menace à l'ordre public $[\ldots]$; que, dans ces conditions, et compte tenu du contexte marqué par une recrudescence des actes antisémites, le ministre de l'intérieur n'a pas commis d'illégalité manifeste en estimant, au vu de l'ensemble des éléments [...] qu'ils caractérisent, que la présence de l'intéressé en France constituait objectivement une menace grave pour l'ordre public $[\ldots]^{6}$.
La solution retenue par le juge du Palais-Royal n'a pourtant pas fait l'unanimité au sein de la doctrine. Il a ainsi été reproché à l'arrêt

[...] à quel point détacher la notion de «menace grave» de celle de condamnation pénale laisse à l'Administration une marge d'appréciation perméable au contexte social, politique ou médiatique ${ }^{7}$.

Et l'annotatrice de poursuivre:

Les faits de l'espèce montrent parfaitement comment la «menace» peut cependant être constituée, du moins dans un examen de l'illégalité manifeste de la décision, par de simples suspicions portant sur une menace future. L'expulsion est alors une mesure sanctionnant de purs soupçons, là où son usage habituel en fait presque une peine complémentaire ${ }^{8}$.

Ces critiques semblent néanmoins assez sévères, et révèlent un désaccord de fond avec l'orientation générale prise par le droit de l'expulsion. Elles ne doivent en tout cas pas masquer la parfaite orthodoxie juridique du raisonnement du Conseil d'État.

1. De manière générale, tout d'abord, l'expulsion est qualifiée traditionnellement de mesure de police administrative. De nature essentiellement préventive, la police administrative a pour objet de sauvegarder l'ordre public, en prévenant les atteintes qui pourraient lui être portées. Et ce n'est pas la rédaction de l'article L. 521-1 du CESEDA qui nous dissuadera de qualifier l'expulsion ainsi :

Sous réserve des dispositions des articles L. 521-2, L. 521-3 et L. 521-4, l'expulsion peut être prononcée si la présence en France d'un étranger constitue une menace grave pour l'ordre public.

Réitérant en cela une position commune du Conseil Constitutionnel et du Conseil d'État, le juge des référés rappelle d'ailleurs dans cet arrêt que:

[...] l'autorité compétente pour prononcer une telle mesure de police administrative, qui a pour objet de prévenir les atteintes à l'ordre public qui pourraient résulter du maintien d'un étranger sur le territoire français, doit caractériser l'existence d'une menace grave au vu du comportement de l'intéressé et des risques objectifs que celui-ci fait peser sur l'ordre public $[\ldots]^{9}$.

En effet, depuis l'arrêt Elfenzi, «l'expulsion d'un étranger n'a pas le caractère d'une sanction, mais d'une mesure de police exclusivement destinée à protéger l'ordre et la sécurité publics ${ }^{10}$. Le Conseil Constitutionnel va dans le même sens et reconnaît que:

4. Voir A. Dencour, E. Andres, «La France crispée sur son ordre public», Plein droit, n 49, 2001, p. 24-27.

5. CE, réf., 7 mai 2015, M. B., nº 389959: L. Carayon, «Menace à l'ordre public et expulsion d'un ressortissant étranger souffrant de troubles psychiatriques: quand le soupçon prévaut sur la protection ", Revue de droit sanitaire et social, 2015, p. 843-852.

6. CE, réf., 7 mai 2015, M. B., cons. 5 .

7. L. Carayon, «Menace à l'ordre public...», p. 845 .

8. Ibid., p. 846 .

9. CE, réf., 7 mai 2015, M. B., cons. 4 .

10. CE, 20 janvier 1988, Ministre de l'Intérieur c. Elfenzi, Recueil Lebon, p. 17 : L'actualité juridique. Droit administratif, 1988, p. 223, concl. C. Vigouroux; Recueil Dalloz, 1989, sommaires, p. 117, obs. P. Wacquet et F. Julien-Laferrière; confirmé par CE, 19 juin 1992, M. Adjim, nº 114605. 
[...] les mesures d'expulsion sont des mesures de police auxquelles sont assignés des objectifs différents de ceux de la répression pénale; qu'aucune disposition de la Constitution, non plus qu'aucun principe de valeur constitutionnelle ne fait obstacle à ce que la loi confère à l'autorité administrative le pouvoir de prendre un arrêté d'expulsion fondé sur des faits de nature à justifier une condamnation pénale, alors même qu'aucune condamnation définitive n'aurait été prononcée par l'autorité judiciaire $[\ldots]^{11}$.

Il faut bien reconnaître que cette position n'est pas sans conséquence quant aux garanties procédurales offertes aux étrangers frappés d'une telle mesure. Ces décisions n'entrent ainsi pas dans le champ d'application de l'article 9 de la Déclaration des droits de l'homme et du citoyen de 1789 , dès lors qu'il n'a pas été porté atteinte aux garanties juridictionnelles de droit commun ${ }^{12}$. De même, il résulte de ce caractère de police administrative l'application immédiate de toute législation nouvelle relative à l'expulsion ${ }^{13}$, et partant, l'inapplicabilité de l'article 7 de la Convention européenne des droits de l'homme ${ }^{14}$.

Sans doute dommageable sur le plan des garanties individuelles ${ }^{15}$, cette position unanime des juridictions nous semble pourtant juridiquement irréprochable. Pour emprunter un vocable usité par les pénalistes, il semble d'ailleurs tentant d'analyser l'expulsion en une mesure de "sûreté» ${ }^{16}$. "Orientée vers l'avenir», cette mesure "ne vise qu'à éviter la commission d'infraction ou la récidive et à protéger la collectivité ${ }^{17}$. Ce ne sont pas les arguments invoqués à l'encontre de cette qualification qui dissuaderont de mener cette analyse. D'une part, le fait que l'expulsion ne soit pas prononcée par un juge ne suffira pas à lui dénier le qualificatif de mesure de sûreté puisque certaines peuvent être prises par l'autorité administrative, telles que les soins psychiatriques sur décision du représentant de l'État dans le département ${ }^{18}$ ou la suspension du permis de conduire ${ }^{19}$. La matière spécifique du droit des étrangers ne nous semble d'ailleurs pas rétive à cette idée de mesure de sûreté. Le placement en rétention administrative ${ }^{20}$ pourrait être appréhendé ainsi en ce sens qu'il vise à rendre plus efficaces et effectives les mesures d'éloignement ${ }^{21}$. D'autre part, l'expulsion n'est pas systématiquement prononcée alors qu'une infraction a déjà été commise. Cet argument est loin d'être décisif à nos yeux. Les «mesures de sûreté» ont en effet pour but «la protection de l'ordre social grâce à la neutralisation des individus considérés comme dangereux ${ }^{22}$. Elle «a, par nature, vocation à intervenir ante delictum ${ }^{23}$, telles les mesures de désintoxication prises, avant toute infraction, à l'encontre des alcooliques dangereux ${ }^{24}$ pour autrui ${ }^{25}$.

2. En cohérence avec son caractère de mesure de police administrative, la jurisprudence sur l'expulsion confirme la nature essentiellement préventive de l'expulsion. Souvent, il est vrai, l'expulsion est prononcée suite à la commission d'infractions assez graves: meurtre ${ }^{26}$, tentative d'assassinat ${ }^{27}$, viol ${ }^{28}$, proxénétisme ${ }^{29}$, coups et blessures volontaires ${ }^{30}$, trafic de stupéfiants ${ }^{31}$, transport d'armes ${ }^{32}$, ou bien encore occupation de locaux avec séquestration de personnes ${ }^{33}$.

11. CC, déc. $\mathrm{n}^{\circ}$ 79-109 DC du 9 janvier 1980, Loi relative à la prévention de l’immigration clandestine..., cons. $6:$ Revue du droit public, 1980, p. 1631, note L. Favoreu; Recueil Dalloz, 1980, jurispr., p. 420, note F. Hamon; L'actualité juridique. Droit administratif, 1980, p. 356, note C. Franck; Recueil Dalloz, 1980, jurispr., p. 249, note J.-B. Auby; Revue générale de droit international public, 1980, p.31, note D. Turpin; Revue administrative, 1980, p. 363, note J.-Y. Vincent; Gazette du Palais, 1980, 1, 258, note L. Hamon.

12. CC, déc. no 93-325 DC du 13 août 1993, Loi relative à la maîtrise de l'immigration et aux conditions d'entrée, d'accueil et de séjour des étrangers en France: Revue française de droit constitutionnel, 1993, p. 587, note L. Favoreu; Revue du droit public, 1994, p. 5, note F. Luchaire et p. 1165, note V. Fabre-Alibert; Droit social, 1994, p. 69, note J.-J. Dupeyroux et X. Prétot; Les petites affiches, 9 septembre 1994, note B. Mathieu et M. Verpeaux; Revue française de droit administratif, 1993, p. 871, note B. Genevois; La semaine juridique, éd. G, 1994, I, 3728, obs. N. Guimezanes; Revue critique de droit international public, 1994, 1, obs. D. Turpin.

13. CE, 30 juillet 1997, M. Abaidia, ${ }^{\circ} 163710$.

14. CE, 24 mars 1997, M. Errahali, no 162318 .

15. En ce sens, voir L. Carayon, «Menace à l'ordre public...».

16. Pour une présentation de la théorie générale des «mesures de sûreté», voir notamment F. Desportes, F. Le Gunehec, Droit pénal général, $15^{\mathrm{e}}$ éd., Paris, Economica, 2008, spéc. p. 1081-1083; J. Pradel, Droit pénal général, $20^{\mathrm{e}}$ éd., Paris, Cujas, 2014, spéc. p. 518-530; B. Bouloc, Droit pénal général, $22^{\mathrm{e}}$ éd. Paris, Dalloz, 2011, spéc. p. 422-434. Certains auteurs opinent cependant dans le sens de l'unification de la peine et de la mesure de sûreté: R. Merle, A. Vitu, Traité de droit criminel, t. I, Problèmes généraux de la science criminelle, droit pénal général, 7 éd., Paris, Cujas, 1997, spéc. p. 826-836.

17. N. Guimezanes, «Réflexions sur l'expulsion des étrangers», in Mélanges dédiés à Dominique Holleaux, Paris, Litec, 1990, p. 185.

18. Ibid., p. 186; Code de la santé publique, art. L. 3213-1 sq.

19. Code de la route, art. L. 224-7.

20. CESEDA, livre V, titres V et VI, art. L. 551-1 à L. 561-2.

21. Voir D. Turpin, «Reconduite à la frontière», in Répertoire de droit international, D. Carreau, P. Lagarde, H. Synvet (dir.), Paris, Dalloz, en ligne, $\$ 43$. Voir, dans le même sens, J.-P. Céré, «Peine (nature et prononcé)», in Répertoire de droit pénal et de procédure pénale, Y. Mayaud (dir.), Paris, Dalloz, en ligne, $\$ 41$.

22. J.-P. Céré, «Peine (nature et prononcé)», $\$ 29$.

23. Ibid., $\$ 37$.

24. Code de la santé publique, ancien art. L. 355-2 sq., aujourd'hui abrogé.

25. Exemple cité par J.-P. Céré, «Peine (nature et prononcé)», \$37.

26. CE, 11 juin 1993, Ministre de l'Intérieur c. $M^{m e}$ Bourkia, no 121424.

27. CE, 2 novembre 1992, Ministre de l'Intérieur c. Ribero, $\mathrm{n}^{\circ} 121715$.

28. CE, 25 juillet 1980, Touami Ben Abdelslem, Recueil Lebon, p. 820.

29. CE, 2 novembre 1979, Ministre de l'Intérieur c. Makalé, Recueil Lebon, p. 823.

30. CE, 26 février 1992, M. Maatar, n 105013; CE, 24 janvier 1994, M. M’Barki, Recueil Lebon, p. 28.

31. CE, 24 mai 1985, Allaf, Recueil Lebon, p. 711.

32. CE, 30 novembre 1984, Di Vincenzo, Recueil Lebon, p. 692.

33. CE, 14 mars 1980, Traore, Recueil Lebon, p. 821 . 
Cela ne doit néanmoins par dissuader de penser l'expulsion dans son essence préventive. Même dans ces cas, l'expulsion est prononcée non point pour sanctionner une seconde fois l'auteur de l'infraction, mais uniquement pour prévenir la commission d'une nouvelle infraction, lorsque l'ensemble du dossier laisse à craindre que la personne récidive dans son comportement infractionnel. Il faut du reste bien noter l'indépendance qui existe entre l'infraction et la mesure d'expulsion. C'est précisément ce qui explique que des infractions, bien qu'étant amnistiées, ont pu donner lieu à la prise d'une telle mesure ${ }^{34}$. Il en va d'ailleurs de même lorsque la personne a déjà purgé sa peine ${ }^{35}$. Les exemples pourraient encore se multiplier, mais ils convergent tous vers un seul point: ce que l'expulsion vient «sanctionner», c'est la dangerosité de la personne.

Ce n'est alors nullement un hasard si le Conseil d'État affirme de manière constante que:

[...] les infractions pénales commises par un étranger ne sauraient, à elles seules, justifier légalement une mesure d'expulsion et ne dispensent en aucun cas l'autorité compétente d'examiner, d'après l'ensemble des circonstances de l'affaire, si la présence de l'intéressé sur le territoire français est de nature à constituer une menace pour l'ordre et la sécurité publics $[\ldots]^{36}$.

Or, ce n'est autre que ce que le Conseil d'État juge en l'espèce. Le rappel des faits opérés montre ainsi que l'intéressé souffre «d'une grave schizophrénie paranoïde qui suscite chez lui des comportements obsessionnels et des crises de délire ${ }^{37}$. Cette pathologie s'est d'ailleurs traduite par la présence injustifiée de l'intéressé auprès de plusieurs lieux appartenant à la communauté juive (il a tenté de s'introduire dans une synagogue, puis à l'intérieur d'une école juive et a également été vu à plusieurs reprises aux abords de synagogues), ainsi que par son comportement violent à l'égard d'agents de la force publique. Sa sœur a d'ailleurs exprimé des craintes concernant la radicalisation de son frère (bien qu'elle soit revenue par la suite sur ses déclarations).

La présente ordonnance du Conseil d'État peut d'ailleurs se réclamer d'un certain nombre de précédents, dans lesquels la mesure d'expulsion avait été confirmée alors qu'aucune infraction grave n'avait encore été commise. Dans cette logique, le ministre de l'Intérieur a légalement pu se fonder sur les liens entretenus par la personne «avec les responsables d'organisations terroristes impliquées dans les attentats meurtriers commis à Paris quelques mois auparavant, et sur le soutien qu'il apportait à ces organisations ${ }^{38}$. Il en va de même pour des «liens réguliers entretenus [...] avec plusieurs organisations terroristes qui projetaient des actions violentes notamment à l'encontre de la communauté française $»^{39}$.

Et il convient bien de noter que dans aucune de ces affaires le juge administratif n'a mentionné de condamnation pénale préalable. Les solutions ont à chaque fois été justifiées par le danger que faisaient peser les intéressés sur la société. Certes, dans ces affaires, le danger avait un caractère d'imminence plus marqué que dans l'arrêt commenté. Les liens avec lesdites organisations étant déjà largement noués, voire, dans certaines des espèces, un attentat terroriste avait déjà été commis. Ces différences factuelles ne doivent pourtant pas laisser à penser que l'analogie avec notre affaire est mal fondée. D'une part, dans l'article L. 521-1 du CESEDA, il n'est pas question d'une «imminence» de la menace, il suffit que celle-ci soit grave. D'autre part, les faits de notre affaire, tels qu'ils ont été rapportés, par des notes blanches ${ }^{40}$ et des témoignages, semblent attester pareillement de la dangerosité de la personne, et montrent en outre sa possible radicalisation.

Une dernière question se posait alors: la pathologie dont souffre l'intéressé, et qui a justifié son expulsion, n'est-elle pas de nature à faire échec à cette mesure, en application du $5^{\circ}$ de l'article L. 521-3 du CESEDA? Ce dernier dispose en effet que:

\begin{abstract}
Ne peuvent faire l'objet d'une mesure d'expulsion qu'en cas de comportements de nature à porter atteinte aux intérêts fondamentaux de l'État, ou liés à des activités à caractère terroriste, ou constituant des actes de provocation explicite et délibérée à la discrimination, à la haine ou à la violence contre une personne déterminée ou un groupe de personnes: [...] $5^{\circ}$ L'étranger résidant habituellement en France dont l'état de santé nécessite une prise en charge médicale dont le défaut pourrait entraîner pour lui des conséquences d'une exceptionnelle gravité, sous réserve de l'absence d'un traitement approprié dans le pays de renvoi [...].
\end{abstract}

34. CE, 27 octobre 1993, M. Stangret, $\mathrm{n}^{\circ}$ 126320; CAA Douai, 10 février 2005, Degoulhd, nº 03 DAoo270.

35. CE, 26 février 1992, M. Yahiaoui, no 125651.

36. CE, 24 janvier 1994, M. M'Barki, Recueil Lebon, p. 28.

37. CE, réf., 7 mai 2015, M. B., cons. 5.

38. CE, 8 juillet 1991, Faker, Recueil Lebon, p. 275; même solution dans CE, 9 octobre 1987, M. X., n 67301; CE, 9 juin 1989 , Ministre de l'Intérieur c. M. Baghat, nº 91821; CE, 8 juillet 1991, Ministre de l'Intérieur c. Uriarte Diaz de Guérenu, Recueil Lebon, p. 197.

39. CE, 29 décembre $1997, M . Z ., \mathrm{n}^{\circ} 168042$

40. Ces «notes blanches», bien qu'étant émises par les services de renseignement sans mention de leur origine, du service dont elles proviennent ou du nom du fonctionnaire les ayant rédigées, se sont vues conférer valeur probante par les juridictions administratives. Voir, en ce sens, CE, Ass., 11 octobre 1991, M. Diouri, Recueil Lebon, p. 941; CE, 22 juin 1994, El Merhebi, nº 12816o. Plus récemment, voir également CAA Paris, 6 mai 2015, M. A., $\mathrm{n}^{\circ}{ }_{14}$ PAo2242; CE, ord., 11 décembre 2015, M. A. B., $\mathrm{n}^{\circ} 394993$, cons. 19. Il est néanmoins permis de s'interroger sur cette solution, bien que solidement ancrée en jurisprudence. En effet, dès 2002, les ministres de l'Intérieur ont affirmé leur intention de bannir cette pratique dans leur service. Dominique de Villepin, en réponse à un sénateur, affirmait en ce sens: «Il n'est pas acceptable en effet dans notre République que des notes puissent faire foi alors qu'elles ne portent pas de mention d'origine et que leur fiabilité ne fait l'objet d'aucune évaluation " (JORF Sénat, 4 juin 2004, p. 3819). Voir également une réponse de Michèle Alliot-Marie (JORF Sénat, 8 novembre 2007, p. 2042). En dernier lieu, Isabelle Attard (députée) a invité le ministre de l'Intérieur Bernard Cazeneuve à se prononcer sur cette question (aucune réponse n'étant pour l'heure publiée) : JORF Assemblée nationale, 5 janvier 2016, question nº 92304, p. 18. 
Le Conseil n'a pas fait droit à cette argumentation; il résultait en effet de l'avis du médecin de l'Agence régionale de santé que l'intéressé pouvait bénéficier d'un traitement approprié dans son pays d'origine ${ }^{41}$.

Tout à fait classique lorsqu'il est question de l'expulsion d'un étranger atteint de troubles psychiques graves ${ }^{42}$, il ne doit pas faire perdre de vue que l'intérêt de cet arrêt est ailleurs. Saisi de l'expulsion d'un «fou », le juge administratif entend prévenir la menace à l'ordre public (en confirmant l'expulsion), plutôt que de laisser le juge pénal la punir une fois que l'infraction a eu lieu. Il n'est pas question ici de discuter du bien-fondé politique de cette posture, simplement de réaffirmer son caractère solidement établi en jurisprudence.

\section{B. L'applicabilité du Code de l'entrée et du séjour des étrangers et du droit d'asile face à un accord bilatéral: faire enfin dire à l'accord ce qu'il dit...}

Faire dire à un accord bilatéral ce qu'il ne dit pas, faire produire à la hiérarchie des normes des conséquences qu'elle ne saurait avoir, telle est «l'aventure» dans laquelle s'est trop souvent engagé le juge administratif lorsqu'il est saisi de la question de l'applicabilité du CESEDA face à un tel accord. C'est précisément dans cette "aventure» que le Conseil d'État s'est lancé lorsqu'il a affirmé, sous des justifications assez nébuleuses, que:

[...] l'accord franco-algérien du 27 décembre 1968 [...] régit de manière complète les règles concernant la nature des titres de séjour pouvant être délivrés aux ressortissants algériens ${ }^{43}$.

De la sorte, les dispositions du CESEDA régissant l'octroi des titres de séjour deviennent inapplicables à ces ressortissants. Si dans certaines hypothèses il est clair que les conséquences sont plus théoriques que pratiques, notamment parce que l'accord de 1968 contient un titre de séjour délivré dans des conditions analogues à son pendant du CESEDA, les conséquences peuvent être nettement plus drastiques en l'absence de telles dispositions dans l'accord. Ainsi, très concrètement, les ressortissantes algériennes victimes de proxénétisme ou de traite des êtres humains ne pourront pas obtenir la carte de séjour temporaire prévue à l'article L. 316-1 du CESEDA ${ }^{44}$.

Ces solutions sont juridiquement regrettables. Déjà,

[...] elle fait une application «à géométrie variable» de la hiérarchie des normes. Au lieu de faire prévaloir la norme internationale sur la loi interne en cas d'incompatibilité de la seconde avec la première, elle consiste à donner un contenu à l'accord - son prétendu caractère exhaustif pour juger contraire à cette "norme supposée», toute disposition plus favorable du CESEDA ${ }^{45}$.

La cohérence même de cette jurisprudence se laisse d'ailleurs difficilement saisir puisque celle-ci continue de réserver le cas des dispositions de procédures du CESEDA, «qui s'appliquent à tous les étrangers en ce qui concerne la délivrance, le renouvellement ou le refus de titres de séjour ", sauf lorsque l'accord de 1968 en dispose (expressément) autrement ${ }^{46}$. Cette distinction entre le fond et la forme laisse largement dubitatif. Pourquoi considérer que la complétude de l'accord franco-algérien s'arrête au fond (la délivrance de la carte), en ne s'étendant pas à la forme (la procédure suivie dans la prise de décision) ${ }^{47}$ ?

Cette tendance à distinguer ce qui est similaire ${ }^{48}$ a pris d'autant plus d'ampleur que la cour administrative d'appel de Marseille admet que cette règle de la complétude de l'accord

[...] ne f[ai]t [...] pas obstacle, en l'absence de dispositions incompatibles expresses, à ce que les ressortissants algériens, en leur qualité de conjoint de citoyens de l'Union européenne, se prévalent des dispositions précitées des articles L. 121-1 et L. 121-3 du Code de l'entrée et du séjour des étrangers et du droit d'asile qui assurent la transposition en droit interne, de la directive 2004/38/CE [... $]^{49}$.

Se situant dans la lignée de l'arrêt Larachi du Conseil d'État ${ }^{50}$, il paraît pour le moins surprenant que les ressortissants algériens victimes de traite ne puissent bénéficier de cette jurisprudence, dans la mesure où l'article L. 316-1 répond lui-même à des exigences communautaires ${ }^{51}$.

Il en va néanmoins différemment lorsque l'accord bilatéral lui-même prévoit qu'il se substitue au CESEDA.

41. CE, réf., 7 mai $2015, M$. B., cons. 7 .

42. CE, 2 juin 2003, $M^{m e} X ., \mathrm{n}^{\circ} 249489$; CE, 22 avril 2005, M. X., nº 270794

43. TA Toulouse, 19 décembre 2007, $M^{m e}$ D., épouse R., nº 0704288: L'actualité juridique. Droit administratif, 2008, p. 995, note C. Sevely-Fournié; TA Cergy-Pontoise, 26 novembre 2009, $M^{m e}$ Fatima Zohra S., nº 0903748: L'actualité juridique. Droit administratif, 2010, p. 502, note E. Costa. Voir également, pour l'applicabilité de l'article L. 313-14: CAA Lyon, $1^{\text {er }}$ juillet 2008, Benhalis, no o7LYo2958.

44. CAA Marseille, 11 juin 2009, M. M’hamed Mezili, nº 07MA04517; CAA Paris, 26 avril 2012, Brahim A., nº 11 PAoo7o8.

45. G. Dujardin, «La protection par le droit des étrangers des victimes de proxénétisme: deux fondements pour un seul régime», Revue du droit public, n' 2, mars 2016, p. 467; voir, dans le même sens, L'actualité juridique. Droit administratif, 2008, note C. Sévely-Fournié, spéc. p. 998.

46. CE, 14 avril 1999, Ministre de l'Intérieur c. Ijqa, Recueil Lebon, tables, p. 820 ; de même dans TA Toulouse, 19 décembre $2007, M^{m e}$ D., épouse R., précité.

47. Voir G. Dujardin, «La protection par le droit des étrangers des victimes de proxénétisme...»; L'actualité juridique. Droit administratif, 20o8, note C. Sévely-Fournié, spéc. p. 998-999.

48. Voir G. Dujardin, «La protection par le droit des étrangers des victimes de proxénétisme...».

49. CAA Marseille, 9 juin 2011, Préfet des Bouches-du-Rhône c. $M^{m e}$ Mehnane épouse Marir, nº9MAo4680: L'actualité juridique. Droit administratif, 2011, p. 1486, note L. Marcovici.

50. CE, 22 mai 1992, Larachi, Recueil Lebon, p. 203: Revue du droit public, 1992, p. 1793, concl. R. Abraham.

51. Voir G. Dujardin, «La protection par le droit des étrangers des victimes de proxénétisme...». 
Dans un avis rendu le 9 novembre $2015^{52}$, la haute juridiction était saisie de la question de l'applicabilité de l'article L. 313-14 à un ressortissant sénégalais. En l'espèce, l'intéressé s'était vu refuser par le préfet de Seine-SaintDenis la délivrance d'une carte de séjour temporaire sur le fondement de l'article L. 313-14 du CESEDA, ce dernier accompagnant sa décision d'une obligation de quitter le territoire français. Saisi d'une requête visant à faire annuler ledit arrêté, le tribunal administratif de Montreuil rejette ces prétentions; la cour administrative d'appel de Versailles, avant de statuer sur l'appel formé contre ledit jugement, décide alors de poser une question au Conseil d'État.

La haute juridiction commence par rappeler (pt. 1) les dispositions de l'article L. 111-2 du CESEDA aux termes duquel «Le présent code régit l'entrée et le séjour des étrangers en France métropolitaine [...] sous réserve des conventions internationales». Appliquant ce principe général - qui au fond découle déjà de l'article 54 de la Constitution $^{53}$-, le juge poursuit en rappelant (pt. 2) les stipulations du paragraphe 42 de l'article 4 de l'accord du 23 septembre $2006^{54}$ :

Un ressortissant sénégalais en situation irrégulière en France peut bénéficier, en application de la législation française, d'une admission exceptionnelle au séjour se traduisant par la délivrance d'une carte de séjour temporaire portant: - soit la mention «salarié» s'il exerce l'un des métiers mentionnés dans la liste figurant en annexe IV de l'Accord et dispose d'une proposition de contrat de travail; / - soit la mention «vie privée et familiale» s'il justifie de motifs humanitaires ou exceptionnels.

Il en découle selon la haute juridiction que «Les stipulations du paragraphe 42 de l'accord du 23 septembre 2006 [...] rendent applicables à ces ressortissants les dispositions de l'article L. 313-14» (pt. 3).

Cette démarche mérite d'être saluée. En creux, on y retrouve en effet un principe sage. Les rapports entre les accords bilatéraux et le Code des étrangers sont régis par les premiers. C'est parce que l'accord prévoit qu'il s'appliquera par dérogation à la législation française qu'il rendra inapplicables les dispositions du CESEDA correspondantes. Ainsi, dans un avis du 2 mars 2012 ${ }^{55}$, le Conseil d'État se fonde sur l'article 11 de l'accord franco-tunisien pour affirmer que l'article L. 313-14 n'est pas applicable s'agissant des ressortissants tunisiens. L'article 11 de l'accord prévoit en effet que:
Les dispositions du présent Accord ne font pas obstacle à l'application de la législation des deux États sur le séjour des étrangers sur tous les points non traités par l'Accord.

Chaque État délivre notamment aux ressortissants de l'autre État tous titres de séjour autres que ceux visés au présent Accord, dans les conditions prévues par sa législation.

Et, dès lors que ledit accord fixe en son article 3 les conditions de délivrance de la carte de séjour «salarié», un travailleur tunisien ne saurait se fonder sur les dispositions de l'article L. 313-14 pour obtenir ladite carte. Comme le rappelle à juste titre cet avis :

Portant sur la délivrance des catégories de cartes de séjour temporaire prévues par les dispositions auxquelles il renvoie, l'article L. 313-14 n'institue pas une catégorie de titres de séjour distincte mais est relatif aux conditions dans lesquelles les étrangers peuvent être admis à séjourner en France, soit au titre de la vie privée et familiale, soit au titre d'une activité salariée.

La haute juridiction a donc mis en place un raisonnement en deux temps: d'abord, elle recherche si l'accord contient une disposition régissant ses rapports avec le droit interne, puis recherche au cas par cas si l'accord régit la délivrance du titre de séjour sollicité. Cette même approche sera d'ailleurs reprise pour les ressortissants marocains ${ }^{56}$, maliens ${ }^{57}$ et béninois ${ }^{58}$. En l'absence de cette disposition "générale», en forme de vade-mecum de l'applicabilité de l'accord, le raisonnement suivi dans l'avis commenté diffère légèrement: c'est l'article 4, $\$ 42$, de l'accord qui renvoie à la législation nationale pour les mesures d'admission exceptionnelle au séjour. Peu importe cette légère différence de raisonnement, qui ne tient qu'à la rédaction de l'accord, il importe surtout de retenir que dans ces hypothèses, le Conseil d'État se « contente» de tirer les conséquences d'affirmations claires d'un accord.

Ce raisonnement peut-il donc s'appliquer à l'accord franco-algérien? Le rapporteur Botteghi a penché pour la négative dans ses conclusions sur l'avis de 2012 :

\footnotetext{
Alors que dans le cas d'accords complets comme l'accord franco-algérien, des décrochages entre le droit commun et les droits applicables aux étrangers couverts par l'accord sont possibles, pour les accords supplétifs, les dispositions de droit commun ont vocation à s'appliquer si elles ne sont pas exclues par les stipulations de l'accord bilatéral $[\ldots]^{59}$.
}

52. CE, avis, 9 novembre 2015, $\mathrm{n}^{\circ}$ 391429: L'actualité juridique. Droit administratif, 2015, p. 2448, concl. X. Domino.

53. Il convient de noter que l'article L. 111-2 est une reprise de l'ordonnance de 1945, époque à laquelle aucune disposition constitutionnelle ne venait trancher explicitement la question des rapports entre le droit interne et le droit international.

54. Tel qu'amendé par un avenant du 25 février 2008. L'accord du 23 septembre et son avenant ont été publiés par le décret nº 2009 -1073 du 26 août 2009.

55. CE, avis, 2 mars 2012, $\mathrm{n}^{\circ} 355208$ : L'actualité juridique. Droit administratif, 2012, p. 1282, concl. D. Botteghi.

56. CE, 31 janvier 2014, Ministre de l'Intérieur c. Nassiri, $\mathrm{n}^{\circ}$ 367306, Recueil Lebon, tables: L'actualité juridique. Droit administratif, 2014, p. 801, note J.-C. Jobart; Recueil Dalloz, 2015, p. 450, obs. O. Boskovic, S. Corneloup, F. Jault-Seseke, N. Joubert, K. Parrot.

57. CE, avis, 7 mai 2013, Dembele, $\mathrm{n}^{\circ}$ 366481, Recueil Lebon, tables, p. 630.

58. CE, avis, 5 juillet 2013, Houeto, $\mathrm{n}^{\circ} 367908$, Recueil Lebon, tables, p. 630.

59. L'actualité juridique. Droit administratif, 2012, p. 1282, concl. D. Botteghi. 
Ce raisonnement se heurte à l'objection citée au début de cette partie: sa prémisse consiste à faire dire à l'accord ce qu'il ne dit pas ${ }^{60}$. Certes, elle pourrait hypothétiquement s'expliquer «par la particularité de la relation historique franco-algérienne ${ }^{61}$. Cette réalité historique a-t-elle pourtant sa place dans les prétoires? Sans doute pas...

Pour conclure cette partie, il serait tout de même de bon ton de préciser que l'impact pratique de ces débats sur l'applicabilité de l'article L. 313-14 doit être largement nuancé. Le Conseil d'État le précise d'ailleurs en creux, à propos de l'inapplicabilité de l'article L. 313-14 aux Algériens:

[...] si l'accord franco-algérien ne prévoit pas, pour sa part, de semblables modalités d'admissions exceptionnelle au séjour, il y a lieu d'observer que ses stipulations n'interdisent pas au préfet de délivrer un certificat de résidence à un ressortissant algérien qui ne remplit pas l'ensemble des conditions auxquelles est subordonnée sa délivrance de plein droit. Il appartient au préfet, dans l'exercice du pouvoir discrétionnaire dont il dispose sur ce point, d'apprécier, compte tenu de l'ensemble des éléments de la situation personnelle de l'intéressé, l'opportunité d'une mesure de régularisation ${ }^{62}$.

Déjà affirmé dans son important avis du 22 août $1996^{63}$ et confirmé dans son remarqué arrêt $M$. Cortes Ortiz ${ }^{64}$, ce pouvoir traditionnel de régularisation des étrangers pourra de même bénéficier à un ressortissant tunisien $^{65}$. Cela se traduira d'ailleurs par une obligation pour le préfet de motiver son refus de procéder à la régularisation ${ }^{66}$.

Quoi qu'il en soit, il est à souhaiter que, de manière générale, le juge administratif fasse preuve de la même sagesse interprétative pour l'accord franco-algérien de 1968 que celle dont il a une nouvelle fois fait preuve dans cet avis de novembre 2015. Il serait grand temps de faire cesser ce paradoxe qui consiste à traiter plus défavorablement un Algérien par rapport aux autres étrangers, y compris ceux qui relèvent d'un accord bilatéral, en dépit de la «relation historique franco-algérienne ${ }^{67}$ sensée leur bénéficier.

\section{Enfin un statut de réfugié solide pour les victimes de traite ${ }^{68}$}

Par une décision très remarquée, la Cour nationale du droit d'asile (CNDA) réitère sa position selon laquelle les victimes de traite des êtres humains (le plus souvent victimes de proxénétisme) peuvent obtenir le statut de réfugié.

Originaire du Nigeria, et contrainte à se prostituer sur le territoire français, la requérante avait sollicité des autorités de l'asile l'octroi d'une protection internationale. D'abord rejetée par l'Office français de protection des réfugiés et des apatrides (OFPRA), la CNDA fit droit à sa demande dans une décision du 29 avril 2011. Elle y affirmait que:

[...] le caractère systématique de ce défaut de protection dans ledit État d'Edo ne doit pas être seulement regardé comme un risque de traitement inhumain et dégradant au sens des dispositions de l'article L. 712-1 du code de l'entrée et du séjour des étrangers et du droit d'asile, mais, s'agissant des prostituées qui sont originaires de cet État et dont il résulte de l'instruction, et notamment des contacts établis avec des associations [...], qu'elles sont à la fois victimes du trafic d'êtres humains susanalysé et désireuses de s'en extraire de manière active, que ces femmes constituent ainsi un groupe dont les membres sont, en raison de ces deux caractéristiques communes qui les définissent, susceptibles d'être exposées à des persécutions au sens des stipulations précitées de l'article 1 A 2 de la Convention de Genève $[\ldots]^{69}$.

Ce raisonnement peinait pourtant à convaincre, en raison de son caractère essentiellement circulaire. En identifiant la persécution - le fait d'être victime de traite des êtres humains - au groupe social dont sont membres ces femmes - le groupe des victimes de traite et désireuses d'en sortir -, la CNDA brise le lien de causalité ${ }^{\circ 0}$ qui doit exister entre la persécution et son motif, aux termes de l'article 1.A.2. Affirmer en substance, ainsi qu'elle le faisait, que la requérante est victime de traite parce qu'elle fait partie du groupe social des victimes de traite avait franchement de quoi surprendre ${ }^{71} \ldots$

60. Voir supra.

61. L'actualité juridique. Droit administratif, 2015, p. 2448, concl. X. Domino.

62. CE, avis, 22 mars 2010, M. Mohamed Saou, n 333679, Recueil Lebon, p. 83: L'actualité juridique. Droit administratif, 2010, p. 583.

63. CE, avis, 22 août 1996, n 359622 : Les grands avis du Conseil d'État, $3^{\mathrm{e}}$ éd., Paris, Dalloz, 2008, p. 345.

64. CE, sect., 4 février 2015, Ministre de l'Intérieur c. M. Cortes Ortiz, $\mathrm{n}^{\circ} 383267$ : L'actualité juridique. Droit administratif, 2015, p. 191, obs. D. Poupeau; Lettre "Actualités Droits-Libertés» de La revue des droits de l'homme, 23 avril 2015, note J. Domenach, en ligne: https://revdh.revues.org/1085; Cahiers de la recherche sur les droits fondamentaux, $\mathrm{n}^{\circ}$ 13, 2015, p. 167, chron. G. Dujardin.

65. CAA Versailles, 20 mai 2010, Boujnah, no 08 VE03829.

66. CAA Paris, 19 décembre 2013, nº 13 PA01926 (à propos d'un Algérien).

67. Pour reprendre l'expression citée plus haut du rapporteur X. Domino.

68. Les idées développées dans cette partie sont, pour leur grande majorité, issues de notre article: G. Dujardin, «La protection par le droit des étrangers des victimes de proxénétisme...».

69. CNDA, 29 avril 2011, $M^{\text {lle }} E$., $\mathrm{n}^{\circ} 10012810$.

70. L'expression «du fait de» utilisée dans l'article 1.A.2 de la Convention de Genève de 1951 implique en effet que la persécution soit liée à l'un des cinq motifs conventionnels. Néanmoins, cette clause se révèle bien ambiguë: est-ce la crainte qui doit être reliée au motif conventionnel ou la persécution elle-même? Si, dans la première hypothèse, la preuve d'une simple causalité suffira, il faudra en revanche dans la seconde hypothèse prouver «une intention discriminatoire spécifique». Voir, en ce sens, A. Korsakoff, «La Convention de Genève revisitée: vers une définition genrée de la persécution", in La loi et le genre: études critiques de droit français, S. Hennette-Vauchez, M. Pichard, D. Roman (dir.), Paris, CNRS éd., 2014, p. 736-753, spéc. p. 746.

71. Cette affirmation, en pratique, peut dans certains cas être nuancée: des femmes qui s'étaient extraites de leur réseau ont pu être contraintes, une fois de retour au pays, de se prostituer de nouveau, notamment en raison de la situation d'extrême précarité matérielle dans laquelle elles se trouvaient. 
Saisi de cette affaire par l'OFPRA, le Conseil d'État censura pour erreur de droit l'arrêt de la CNDA de 2011, au motif que la juridiction de l'asile n'avait pas caractérisé l'existence d'un groupe social:

[...] en jugeant que les femmes victimes de réseaux de trafic d'êtres humains et ayant activement cherché à échapper à leur emprise constituaient un groupe social sans rechercher si, au-delà des réseaux de proxénétisme les menaçant, la société environnante ou les institutions les percevaient comme ayant une identité propre, constitutive d'un groupe social au sens de la convention, la Cour nationale du droit d'asile a commis une erreur de droit $[\ldots]^{72}$.

Pour enfin franchir solidement ce pas de l'octroi d'un statut de réfugié aux victimes de traite, il était donc vital pour la Cour de s'extraire des deux écueils dénoncés plus haut. Il fallait pour elle caractériser l'existence d'un «certain groupe social» au sens de l'article 10, $\S_{1}$, d, de la directive 2011/95/UE ${ }^{73}$, et se sortir de ce raisonnement circulaire, intellectuellement bien peu satisfaisant.

C'est de ce double écueil que la Cour est donc parvenue à s'échapper dans son arrêt $M^{\text {lle }} J o y$ A. B. du 24 mars $2015^{74}$.

S'agissant tout d'abord de l'appartenance à un «certain groupe social» de la requérante, la Cour indique

[...] qu'en raison de leur soumission à un système de traite [...] marquée à leur entrée dans un réseau de proxénétisme par une cérémonie rituelle traditionnelle [...], des années d'exploitation dont elles ont été victimes en Europe, puis des démarches qu'elles engagent en vue de s'extraire [...], les femmes qui ont été soumises à un réseau de trafic d'êtres humains et qui tentent d'échapper à l'emprise de celui-ci doivent être considérées comme partageant une histoire commune $[\ldots]$.

Et la Cour de poursuivre

[...] qu'en raison notamment de la cérémonie vaudou imposée aux jeunes femmes [...] et notamment du serment que sont tenues de prêter les victimes qui scelle leur soumission, une distanciation d'avec le réseau, et donc la remise en cause de ce serment, les expose à un regard réprobateur de la part [...] de la société environnante [...].

Ce faisant, la Cour a caractérisé les deux éléments nécessaires à la reconnaissance d'un certain groupe social.
L'article 10 de la directive 2011/95/UE (précité) pose en effet deux conditions cumulatives pour que ledit groupe soit reconnu. Il faut d'une part qu'il soit caractérisé par une histoire commune (comme c'était le cas en l'espèce), une caractéristique innée, ou encore une croyance à ce point essentielle qu'il ne devrait pas être exigé de la personne qu'elle y renonce. Et, d'autre part, il importe que ce groupe soit perçu comme différent par la société environnante. Or, contrairement à ce que deux arrêts d'assemblée du Conseil d'État de $2012^{75}$ auraient pu laisser à penser, ces deux conditions apparaissent bel et bien comme cumulatives. Il aurait d'ailleurs pu en aller autrement dans la mesure où l'article 3 de la directive 2011/95/UE admet qu'une norme interne soit plus favorable à condition qu'elle ne soit pas contraire aux dispositions de la directive ${ }^{76}$. Notons enfin sur ce point que la reconnaissance d'un groupe social pour les victimes de traite originaires de l'État d'Edo au Nigeria est nouvelle. Hormis l'arrêt de 2011 cassé par le Conseil d'État en 2013 dans cette affaire, la Cour avait jusque-là systématiquement refusé d'accorder le statut de réfugié à ces femmes ${ }^{77}$, tout en en ayant déjà caractérisé l'existence pour d'autres nationalités ${ }^{78}$.

S'agissant ensuite de l'écueil du raisonnement circulaire, la Cour, pour y échapper, développe ce qu'on pourrait appeler une théorie de la "persécution à double détente». Pour ce faire, dans un considérant aux forts accents d'obiter dictum, elle réaffirme solennellement que «la traite des êtres humains, qui est condamnée, dans ses diverses modalités, [...] constitue une persécution ». Mais, et c'est là que réside la nouveauté, c'est une autre persécution, qu'on serait tenté de qualifier de "dérivée», qui est finalement retenue aux fins d'octroyer le statut de réfugié. À cette fin, la juridiction de l'asile retient en l'espèce qu'en cas de retour dans son État d'origine l'intéressée s'exposerait aux mesures répressives engagées par le temple Ayelala dans lequel elle a prêté serment de rembourser sa dette. Il est de même relevé que les acteurs des réseaux de proxénétisme exercent également de graves pressions, pouvant aller dans certains cas jusqu'à l'assassinat de la personne elle-même ou d'un membre de sa famille. Enfin, la Cour mentionne les graves discriminations dont la personne fera l'objet en cas de retour dans son État d'origine: celles-ci occasionneront «des

72. CE, sect., 25 juillet 2013, OFPRA c. $M^{\text {me }}$ C. A. B., $\mathrm{n}^{\mathrm{o}} 350661$, cons. 5

73. Directive 2011/95/UE du Parlement européen et du Conseil du 13 décembre 2011 concernant les normes relatives aux conditions que doivent remplir les ressortissants des pays tiers ou les apatrides pour pouvoir bénéficier d'une protection internationale, à un statut uniforme pour les réfugiés ou les personnes pouvant bénéficier de la protection subsidiaire, et au contenu de cette protection.

74. CNDA, 24 mars 2015, M $M^{\text {Ule }}$ Joy A. B., no 10012810.

75. CE, Ass., 21 décembre 2012, Darbo-Fofana, $\mathrm{n}^{\circ}$ 332491; CE, Ass., 21 décembre 2012, Fofana, $\mathrm{n}^{\circ} 332492$. La haute juridiction y inaugure un considérant de principe: «Un groupe social [...] est constitué de personnes partageant un caractère inné, une histoire commune ou une caractéristique essentielle à leur identité et à leur conscience, auxquels il ne peut leur être demandé de renoncer, ou une identité propre perçue comme étant différente par la société environnante ou par les institutions; [...] l'appartenance à un tel groupe est un fait social objectif qui ne dépend pas de la manifestation par ses membres, ou, s'ils ne sont pas en mesure de le faire, par leurs proches, de leur appartenance à ce groupe» (nous soulignons). Malgré l'utilisation de la conjonction de coordination «ou», le Conseil d'État considère les deux conditions comme cumulatives. Voir, en ce sens, CE, sect., 25 juillet 2013, OFPRA c. $M^{m e}$ C. A. B.; CE, 20 mars 2013, $\mathrm{n}^{\circ} 339186$.

76. Voir A. Korsakoff, «Les victimes de persécutions de genre, de "nouveaux" réfugiés? Réflexions sur l'étendue de la mobilisation du motif du groupe social», Cahiers de la recherche sur les droits fondamentaux, $\mathrm{n}^{\circ}$ 13, 2015, p. 77-88, spéc. p. 81. Les juridictions anglo-saxonnes ont d'ailleurs fait ce choix: voir les références citées par A. Korsakoff.

77. Voir, en ce sens, CNDA, 3 avril 2012, $M^{\text {He }}$ O., no 11020945 C; CNDA, 13 mars 2012, $M^{\text {me }}$ O., no 11016563 C.

78. Pour une femme kosovare, obligée de se prostituer dans un hôtel de Pristina: CNDA, 15 mars 2012, $M^{m e}$ O., $\mathrm{n}^{\circ}$ 11017758. Pour une femme ukrainienne victime de traite dans son pays: CNDA, 12 juillet 2012, $M^{\text {lle }} S Z, \mathrm{n}^{\circ} 11026228$. 
difficultés certaines à se réintégrer [...], trouver un emploi, se marier et entretenir des relations sociales normales». Cette approche de la "persécution à double détente» nous semble également nouvelle dans sa jurisprudence. Dans les deux arrêts de 2012 dans lesquels la Cour a accordé le statut de réfugié à des victimes de traite, elle est, les deux fois, tombée dans l'écueil du raisonnement circulaire. Si, les deux fois, la Cour avait établi l'existence d'un groupe social, le raisonnement utilisé pour aboutir à l'octroi du statut n'en était pas pour autant satisfaisant.

Ainsi, elle a accordé le statut de réfugié à une femme obligée de se prostituer dans un hôtel de Pristina au motif que:

[...] la soumission de femmes à la prostitution contre leur gré constitue une persécution au sens de l'article $1^{\text {er }}, \mathrm{A}, 2 \mathrm{de}$ la Convention de Genève; que les femmes victimes d'un réseau de prostitution au Kosovo parvenues à s'en échapper doivent être regardées, du fait de cette expérience qui leur est commune et de l'opprobre dont elles font ensuite aujourd'hui l'objet de la part de la société sans pouvoir, dans le contexte sécuritaire actuel prévalant dans ce pays, espérer une action efficace de la part des autorités, comme appartenant à un groupe social au sens des stipulations précitées $[\ldots]^{79}$

Ce raisonnement n'est pourtant pas allé sans susciter certaines réserves au sein de la doctrine. Il lui est ainsi reproché «d'invisibiliser» les «vraies» persécutions ${ }^{80}$. Mais, sans avoir véritablement d'impact pratique négatif, il est guidée par la nécessité d'échapper au raisonnement circulaire dénoncé plus haut. L'attitude de la Cour tranche ici: si elle est effacée du raisonnement «final», la persécution «principale» est rappelée en guise de frontispice de son raisonnement, alors même que ce rappel est juridiquement inutile. Pourquoi? Peut-être pour marquer une certaine gêne face au litige qui lui est soumis...

Rappeler la persécution «principale» lui servirait ainsi à marquer son attachement politique aux instruments de lutte contre la traite, tandis que la persécution "dérivée» serait perçue comme le palliatif à son impossibilité juridique de retenir la première comme "crainte de persécution"

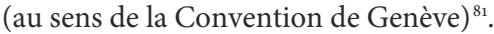

La consistance du groupe social ainsi reconnu appelle également quelques commentaires. De manière générale, le groupe social créé pour les victimes de traite se caracté- rise par deux éléments : le fait d'en avoir été victime, et le fait d'avoir voulu s'extraire du réseau. Le premier ne pose pas réellement de difficulté; le second appelle quelques explications. Sur un plan politique, le fait de limiter le groupe aux seules victimes ayant cherché à s'extraire du réseau se comprend: de même que pour l'article L. 316-1 du CESEDA, le but est de ne pas offrir un titre de séjour à toutes les personnes concernées, pour ne pas compromettre encore un peu plus leur sortie effective du réseau ${ }^{82}$. Sur le plan juridique également, il nous semble se justifier. Il s'inscrit dans un courant jurisprudentiel qui accorde une prédominance aux caractéristiques «ontiques ${ }^{83} \mathrm{du}$ groupe (le fait d'avoir fui quelque chose, le fait d'avoir tenté d'échapper à une situation), plutôt qu'à sa caractéristique "ontologique» ${ }^{84}$. Ainsi, à partir de 2004, ont été reconnus les groupes des «femmes entendant se soustraire aux mutilations génitales féminines ${ }^{85}$, "entendant se soustraire à un mariage imposé ${ }^{86}$, ou bien encore celui des femmes "fuyant des crimes d'honneur ${ }^{87}$. La liste est longue ${ }^{88}$ et montre que, de plus en plus, les victimes ne sont plus protégées pour ce qu'elles sont, mais pour ce qu'elles font ${ }^{89}$. Or, si cette démarche alourdit sans doute la charge de la preuve, elle nous paraît indispensable sur le plan logique (au moins, pour les victimes de traite). Dès lors que la première caractéristique du groupe s'identifie à la persécution «principale», la logique veut que la «crainte de persécution » découle de cette volonté de s'extraire. En clair, c'est parce que la personne a voulu fuir le réseau que ce dernier risquera de lui faire subir des représailles en cas de retour au pays.

Enfin, un élément interroge. Le groupe social reconnu ici paraît différent de celui qui caractérise les victimes de traite à l'étranger. L'arrêt commenté nous parle ainsi des « victimes de la traite des êtres humains [...] qui ont tenté de s'extraire de leur condition », tandis que les arrêts de 2012 (précités), qui se reportaient à des situations de traite commises à l'étranger, mentionnent le groupe des «femmes qui ont été contraintes de se prostituer et sont parvenues à échapper à leurs proxénètes».

Est-ce à dire que la Cour opère une distinction entre la traite des êtres humains et le proxénétisme? Cela nous semble peu plausible, et n'aurait d'ailleurs guère de sens dans l'office de la Cour. Elle n'est pas juge pénal; elle intervient pour apprécier si le demandeur d'asile est victime d'une persécution, et, dans l'affirmative, si celle-ci

79. CNDA, 15 mars 2012, $M^{\text {me }} O$

80. Voir Les cahiers du social, $\mathrm{n}^{\circ}$ 32, 2011, Le droit d'asile au féminin. Cadre législatif et pratique, E. Soulard (dir.), spéc. p. 32-41 («L'interprétation inadaptée des violences faites aux femmes comme constitutives de persécutions ou de menaces graves»); A. Korsakoff, «Les victimes de persécutions de genre...».

81. G. Dujardin, «La protection par le droit des étrangers des victimes de proxénétisme... », p. 462.

82. En pratique, on s'aperçoit que ce sont les réseaux eux-mêmes qui poussent les jeunes femmes à demander l'asile (évidemment en racontant une fausse histoire), afin que leur situation administrative soit régularisée.

83. C'est-à-dire qui se rapporte à l'étant, à un certain comportement du groupe.

84. Autrement dit, caractéristique qui se rapporte exclusivement à ce qu'il est: victime de traite, d'excision, de mariage forcé, etc.

85. CRR, 21 septembre 2004, $M^{\text {lle }} B$., $\mathrm{n}^{\circ} 452011$ (nous soulignons).

86. CRR, 15 octobre 2004, $M^{l l e} N ., \mathrm{n}^{\circ} 444000$ (nous soulignons).

87. CRR, 26 janvier 2006, $M^{\text {lle }}$ S., $\mathrm{n}^{\mathrm{o}} 544746$ (nous soulignons).

88. Pour de nombreux exemples, voir Les cahiers du social, $\mathrm{n}^{\circ}$ 32, 2011, Le droit d'asile au féminin..., p. 44-51.

89. Voir A. Korsakoff, «Les victimes de persécutions de genre...». 
se rattache à l'un des motifs énoncés à l'article 1.A.2 de la Convention de Genève.

Est-ce également à dire que la Cour serait moins exigeante lorsque les faits de traite ont eu lieu en France? Dans ce cas, il suffirait que la personne ait tenté de fuir son réseau, alors que pour des faits de traite à l'étranger il faudrait qu'elle soit parvenue à s'échapper... Pareille conclusion nous paraît largement hasardeuse... Bien que les arrêts aboutissant à l'octroi d'un statut de réfugié soient encore très rares, le caractère ténu de la distinction nous amène à douter du bien-fondé de cette interpré- tation. Cette différence tient donc, probablement aux circonstances propres aux trois arrêts.

Un mot pour finir... L'avenir pratique de cette jurisprudence... Celui-ci nous paraît incertain. L'arsenal offert par le droit commun devient en effet particulièrement riche. En plus de la carte de séjour d'un an offerte aux victimes qui ont porté plainte contre leur réseau, qui sera d'ailleurs délivrée de plein droit ${ }^{90}$, une autorisation provisoire de séjour d'au moins six mois pourra leur être délivrée si, à défaut d'avoir porté plainte, elles sont engagées dans un processus de sortie de la prostitution ${ }^{91}$.

90. Au terme d'un marathon législatif qui a duré plus de deux ans, la loi nº 2016-444 visant à renforcer la lutte contre le système prostitutionnel et à accompagner les personnes prostituées a enfin vu le jour. Son article $8,1^{\circ}$, consacre en effet la délivrance de plein droit de la carte de séjour prévue à la première phrase de l'article L. 316-1 du CESEDA.

91. Le nouvel article L. 316-1-1 disposera ainsi: «Une autorisation provisoire de séjour d'une durée minimale de six mois peut être délivrée, sauf si sa présence constitue une menace pour l'ordre public, à l'étranger victime des infractions prévues aux articles 225-4-1 à 225-4-6 et 225-5 à 225-10 du code pénal qui, ayant cessé l'activité de prostitution, est engagé dans le parcours de sortie de la prostitution et d'insertion sociale et professionnelle mentionné à l'article L. 121-9 du code de l'action sociale et des familles. La condition prévue à l'article L. 313-2 du présent code n'est pas exigée. Cette autorisation provisoire de séjour ouvre droit à l'exercice d'une activité professionnelle. Elle est renouvelée pendant toute la durée du parcours de sortie de la prostitution et d'insertion sociale et professionnelle, sous réserve que les conditions prévues pour sa délivrance continuent d'être satisfaites». 